\title{
Thoracic imaging finding of rheumatic diseases
}

\author{
Maryam Gul ${ }^{1}$, Sadia Moinuddin ${ }^{2}$, Aisha Alam ${ }^{3}$ Iqra Aftab ${ }^{4}$, Zunairah Shah ${ }^{5}$, Ammar Chaudhry ${ }^{6}$ \\ ${ }^{1}$ Department of Rheumatology, Southern California Kaiser Permanente, Lancaster, CA, 93534, USA; ${ }^{2}$ Department of Internal Medicine, San \\ Antonio Regional Medical Center, Upland, CA 91786, USA; ${ }^{3}$ Medical Student, Caribbean Medical University, Willemstad, Curaçao; ${ }^{4}$ Department of \\ Internal Medicine, Maimonides Medical Centre, Brooklyn, NY 11219, USA; 5 Department of Internal Medicine, Louis A Weiss memorial hospital, \\ Chicago, IL 60640, USA; ${ }^{6}$ Department of Radiology, City of Hope National Medical Center, Duarte, CA 91010, USA \\ Contributions: (I) Conception and design: M Gul, S Moinuddin; (II) Administrative support: A Alam; (III) Provision of study materials or patients: I \\ Aftab; (IV) Collection and assembly of data: Z Shah; (V) Data analysis and interpretation: A Chaudhry; (VI) Manuscript writing: All authors; (VII) \\ Final approval of manuscript: All authors. \\ Correspondence to: Maryam Gul, MD. Department of Rheumatology, Southern California Kaiser Permanente, 615 West Ave L, Lancaster, CA, 93534, \\ USA. Email: maryamgul@gmail.com.
}

\begin{abstract}
In the era of Precision Medicine, diagnostic imaging plays a key role in initial diagnosis and treatment response assessment in thoracic manifestation of various rheumatic disorders; resulting in increased dependency on imaging for treatment planning. Chest radiographs serve as a good initial screening tool for assessment of emergent and urgent thoracic conditions, e.g., pneumothorax, pulmonary edema, consolidation and pleural effusions. Cross-sectional imaging techniques, e.g., computed tomography (CT) and positron emission tomography-computed tomography (PET-CT) are most commonly utilized to evaluate more detailed pulmonary and mediastinal manifestations of rheumatic conditions. Magnetic resonance imaging (MRI) and ultrasound are most commonly used in cardiovascular, neural and musculoskeletal structures. This review article aims to highly key common thoracic imaging findings of rheumatic disorders, highlighting imaging test of choice for the particular disorder.
\end{abstract}

Keywords: Rheumatology; radiology; rheumatoid arthritis; granulomatosis with polyangiitis (GPA); microscopic polyangiitis (MPA); giant cell arteritis (GCA); sarcoidosis

Submitted Dec 27, 2019. Accepted for publication Mar 31, 2020.

doi: $10.21037 /$ jtd.2020.04.16

View this article at: http://dx.doi.org/10.21037/jtd.2020.04.16

\section{Introduction}

Improved understanding of pathophysiology of rheumatic disorders has led to successful development of novel targeted therapeutics (e.g., tofacitinib, tocilizumab, TNF inhibitors etc.), which has improved functional outcomes and survival. This growth in precision medicine has been complemented well with advent of novel imaging techniques that can provide high-resolution dynamic point of care in vivo whole-body assessment. This simultaneous growth in novel therapeutics and advanced imaging have led to increased utilization of imaging to accurately diagnose spatial extent of disease in clinical trials as well as routinely clinical practice. For example, in acutely symptomatic patients, chest radiographs are used as screening tools to assess for urgent thoracic conditions, e.g., pneumothorax, pulmonary edema, consolidation, and pleural effusions. Cross-sectional imaging techniques (CT, PET-CT) can more precisely evaluate the aforementioned as well as identify conditions such as pneumonitis, dissection, pulmonary embolism, coronary arterial disease, etc., which may not be adequately assessed on chest radiographs. MRI and ultrasound provide more precise information about the cardiovascular system (ejection fraction, cardiac wall motion, valves, myocardium), nervous system (e.g., spinal cord, nerve roots,) and musculoskeletal structures (joints, myofascial structures). In modern practice, combination of these imaging techniques is routinely used to help establish a diagnosis at point-of-care; especially in scenarios in nonacademic centers where serology and/or biopsy results may 

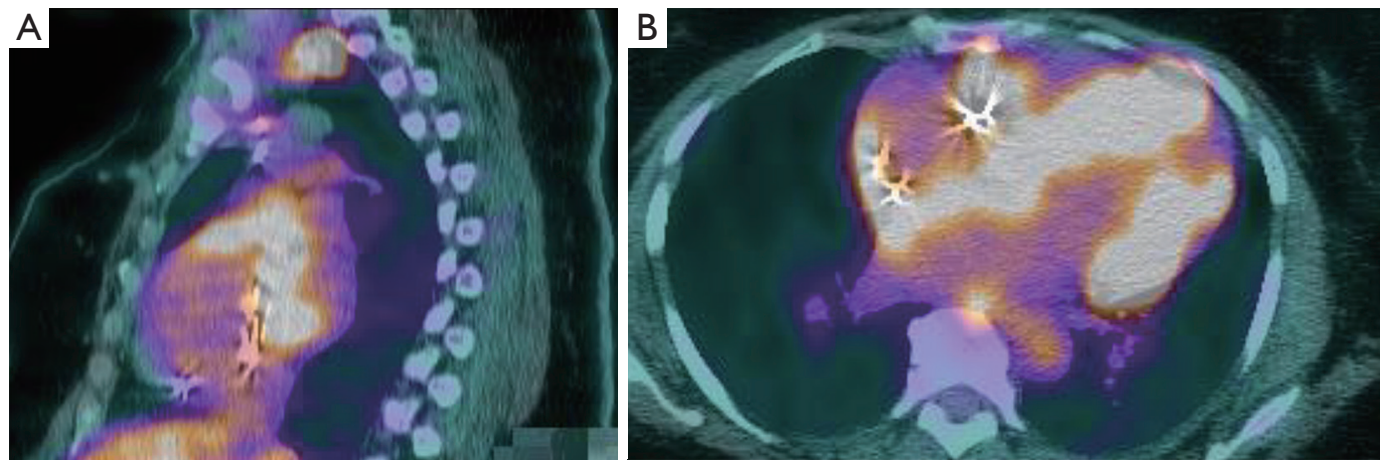

Figure 1 Sagittal (A) and coronal (B) PET-CT images of the thorax demonstrate multichamber cardiomegaly and heterogeneous myocardial wall thickening with corresponding increased FDG uptake. These findings are consistent with cardiac sarcoidosis and were confirmed on biopsy.

take days to result. Imaging is also used to assess for disease progression and treatment response (cure, partial response, no response); all of which play a key role in identifying best treatment strategy for the patient. This review article will focus on thoracic imaging findings of common and uncommon autoimmune diseases including: giant cell arteritis, takayasu arteritis, poly arteritis nodosa, rheumatoid arthritis, granulomatous polyangiitis, eosinophilic granulomatous polyangiitis, microscopic polyangiitis, polymyositis, dermatomyositis, sarcoidosis, Sjogrens syndrome and systemic lupus erythematous.

\section{Sarcoidosis}

Sarcoidosis is an idiopathic autoimmune disorder that results in systemic non-caseating granuloma including lungs, lymph nodes, soft tissues (heart, liver), etc. Approximately $90 \%$ of the patients present with pulmonary and mediastinal involvement with sarcoidosis (1). The reminder can present with skin manifestations, join involvement, eye involvement, neurological signs or cardiac infiltration. This disease is more common in female than males and is more prevalent in the African American population (1). Thoracic manifestation can be asymptomatic in $50 \%$ of the patients. Common respiratory symptoms include cough, dyspnea and shortness of breath (2). Pulmonary function test in advanced stage of the disease show restrictive lung disease pattern with a reduction in DLCO (3). Cardiac sarcoid (Figure 1) can result in pericarditis; or myocardial infiltration, which can cause conduction abnormalities (commonly bradycardia) and heart failure (3).

Although chest X-ray and CT scan can be used to evaluate the initial thoracic manifestations, when available, high-resolution CT is preferred to more accurately diagnose spatial extent of disease. Most common imaging findings include: mediastinal and bilateral hilar lymphadenopathy; interstitial lung disease (usually peribronchovascular or centrilobular micronodules, which can progress to fibrosis); and pleural effusions (3). In sarcoid patients with cardiac dysfunction, MRI or PET-CT are the diagnostic test of choice to assess extent of myocardial infiltration of sarcoidosis (3). Initial treatment for sarcoidosis is high dose steroids, while methotrexate (MTX) can be used as steroid sparing agent. In treatment resistant case, or fulminant disease, cyclophosphamide can be used $(2,3)$.

\section{Giant cell arteritis (GCA)}

GCA is a systemic vasculitis that affects the large and medium-sized arteries. It commonly involves temporal and other cranial arteries affecting people over the age of 50. However, since GCA is a systemic vasculitis, vascular involvement can occur anywhere in the body (4).

The diagnostic criteria per American College of Rheumatology (ACR) requires at least 3 of the following: age $>50$ years, new onset localized headache, temporal artery tenderness or reduced pulsation, elevated erythrocyte sedimentation rate $\geq 50 \mathrm{~mm} /$ hour, and abnormal arterial biopsy (demonstrating vasculitis with predominantly mononuclear cell infiltration, granulomatous inflammation or evidence of giant cells) (5). Temporal artery biopsy should be performed to confirm the diagnosis (5); however, corticosteroids should be initiated as soon as the clinical diagnosis is made due to the high risk of vision loss from 
temporal artery involvement (6). Other symptoms include jaw claudication as well as aches and stiffness affecting the neck, shoulder and pelvic (7).

Thoracic complications include arm claudication or subclavian steal syndrome if the subclavian artery is affected (7). Involvement of the thoracic or abdominal aorta can also cause aortic valve regurgitation, aortic aneurysm and aortic dissection (8). In addition, Giant cell arteritis is associated with increased risk of myocardial infarction, stroke, and peripheral vascular disease (9).

A combination of Fluorodeoxyglucose (FDG) PET-CT can be highly sensitive and moderately specific for diagnosis of giant cell arteritis (6).

In addition, PET-CT and PET-MRI can also help diagnose aortitis (6). These imaging modalities should especially be considered in patients with atypical clinical presentations of giant cell arteritis. In GCA, PET imaging shows accumulations in aorta and great arteries in up to $83 \%$ of patients (6).

GCA is treated with high dose oral prednisone, or IV pulse corticosteroids if vision loss is involved. Patients typically improve dramatically within 5 days (10).

\section{Takayasu arteritis (TA)}

TA is a chronic, large vessel granulomatous disease that predominantly affects the aorta and its branches, leading to luminal stenosis and aneurysms in these vessels (11). It can also affect the coronary and pulmonary arteries (11). This disease is more common in women, particularly in Asian women, and often presents in patients under the age of 40 . Also known as the "pulseless disease", clinical findings in TA may include weak or absent peripheral pulses, arterial bruits, carotidynia, angina, claudication and $>10 \mathrm{mmHg}$ discrepancy in blood pressure between upper limbs (12).

Traditionally, conventional angiography was used as the gold standard for the diagnosis of Takayasu arteritis; however, CT and MR angiography have now emerged as highly accurate diagnostic modalities. The classic manifestation of TA on CT angiography is concentric mural thickening, that is several millimetres wide, of the involved arteries (13). Other imaging options include Positron emission tomography (PET), which in combination with CT (PET-CT) or MR (PET-MR), can be used to evaluate for possible large-vessel vasculitis (13). The "hot" segments of the artery (i.e., those with increased standardized uptake values) can help diagnose large vessel vasculitis (13). Color Doppler ultrasound may also identify early stage blood vessel stenosis (13). Elevated levels of acute phase reactants in the blood can also support the diagnosis but normal levels of erythrocyte sedimentation rate (ESR) and C-reactive protein (CRP) do not rule out the disease (12).

Patients with Takayasu arteritis can suffer from vascular complications, such as aortic regurgitation due to aortic root dilation, or even aortic dissection or rupture (13). They are also prone to cardiac, limb or cerebral ischemia due to luminal narrowing of large blood vessels supplying these regions (13). Involvement of pulmonary artery can also lead to pulmonary hypertension (13).

Treatment of TA involves early initiation of high-dose corticosteroid therapy with gradual tapering (14).

\section{Polyarteritis nodosa (PAN)}

PAN is a systemic, necrotizing vasculitis that typically affects medium sized arteries. It can also affect small sized arteries; however, unlike some small vessel vasculitidies, PAN is not associated with antineutrophil cytoplasmic antibodies (ANCA) or glomerulonephritis $(15,16)$. PAN may either present with systemic symptoms or with localized symptoms involving the skin or the brain.

PAN can be triggered by hepatitis B, hepatitis C, CMV, HIV, parvovirus B19, inflammatory bowel disease and group A streptococcus infections (17).

Systemic symptoms of PAN include skin findings such as erythematous nodules, palpable purpuric lesions or livedo reticularis. Renal involvement can also occur causing renal insufficiency and hypertension (18).

Although uncommon, myocardial infarction may also occur from narrowing of coronary arteries. In addition, patients may experience heart failure from involvement of coronary arteries leading to ischemic cardiomyopathy or from uncontrolled hypertension due to renal disease (19). Patients may also present with GI, neurologic or ophthalmologic symptoms (19).

The confirmatory test for PAN is a biopsy of the clinically affected organ that shows a small or medium sized artery containing polymorphic cells. Visceral angiography may be performed to detect microaneurysms if PAN is suspected but a biopsy cannot be obtained (19).

\section{Polymyositis and dermatomyositis}

Polymyositis (PM) and dermatomyositis (DM) are inflammatory myopathies that present with shared features and clinical manifestations (20). They are characterized by 
increased muscle markers as a reflection of the inflammatory state (21). The presenting muscles involved are generally proximal and symmetrical (21). The primary differentiating features are the heliotrope rash and Gottron's papules seen in DM (21). In clinically amyotrophic DM (CADM), patients have the classic skin findings but no muscle weakness (18). DM is driven by complement deposition, whereas PM is mediated by CD8-positive T-cells (21). In patients diagnosed with either PM or DM, lab findings include elevated CK, AST, ALT, LDH and aldolase (21). The specific antibodies in PM and DM are anti-aminoacyl tRNA-synthetase and anti-melanoma differentiationassociated gene 5(anti-MDA5) (20). To confirm the diagnosis, a muscle biopsy is indicated (20).

The thoracic complications seen in DM and PM extend to the pulmonary, cardiac and gastrointestinal systems. The main pulmonary complication is interstitial lung disease (IDL), seen in $35-40 \%$ of patients $(21,22)$. Due to the high risk of mortality, there is a need for screening and diagnosing IDL (22). In order to do this effectively, upon diagnosis of PM and DM, patients should have high-resolution computed tomography (HRCT) scans and pulmonary function tests done regularly (23). This allows for the best follow-up treatment to be initiated (24). Other pulmonary complications include malignancy, respiratory muscle weakness, pulmonary hypertension and aspiration pneumonia (24). In order to effectively screen for malignancy, patients should have regular CT/PET scans done (25). The cardiac complications are less clinically significant in patients with DM and PM. Patients may present with non-life threatening ECG changes and rarely CHF or valvular abnormalities (23). Alternatively, patients may present with dysphagia to liquids and solids because of muscle tone loss in the pharyngo-esophageal junction (25).

\section{Sjogrens syndrome}

Sjogrens syndrome is an autoimmune disease of the exocrine glands characterized by lymphoplasmacytic infiltration of the salivary and lacrimal gland (26) Patients usually present with dry eyes and dry mouth. Diagnosis is made based on clinical presentation and laboratory findings. Antibody testing may show: positive anti-SSA (anti-Ro) (50-70\% of cases), positive anti-SSB (anti-La) $(50-70 \%$ of cases), positive antinuclear antibodies (50-90\% of cases), and possible positive rheumatoid factor (30-90\% of cases). Labial salivary gland biopsy may be indicated to confirm diagnosis, specifically in patients with negative anti-Ro or
anti-La antibodies. Biopsy demonstrates dense inflammatory infiltrate in exocrine glands (26). At this point, salivary gland scintigraphy or contrast sialography may be useful in evaluation of salivary gland function (26). There is a $5 \%$ prevalence of Sjogren's syndrome in the general population. The disease typically affects females greater than males and hits the age group of 40-60 years old the most (26).

In general, respiratory symptoms are not severe and patients present with dry cough or dyspnea. These manifestations may be seen in patients with and without respiratory symptoms (26).

Pulmonary involvement is not usually characterized on chest $\mathrm{X}$-ray and high resolution computed tomography (CT) is required to detect pulmonary manifestations. The pulmonary manifestations of primary Sjögren syndrome may be categorized as airway abnormalities, interstitial pneumonias, and lymphoproliferative disorders (26). When there is damage to the central and peripheral bronchi and bronchioles, would manifest as bronchiectasis, bronchial wall thickening, and centrilobular nodules and branching nodular opacities (26). Follicular bronchiolitis would be demonstrated by mild thickening of interlobular septa and air cysts (26). Interstitial pneumonias include-nonspecific interstitial pneumonitis usual interstitial pneumonitis (UIP), organizing pneumonia, and LIP (26). They are manifested as ground glass opacities, non-septal linear opacities and interlobular septal thickening, cyst and reticular fibrosis (26).

\section{Microscopic polyangiitis (MPA)}

MPA is an idiopathic autoimmune disease characterized by a systemic vasculitis that predominantly affects the smallcaliber blood vessels, and is associated with the presence of antineutrophil cytoplasmic autoantibodies (ANCA) (27). MPA has a male predominance with an average of onset between 50-60 years old. Most patients present with vague symptoms such as fever and weight loss (27).

Currently there is no lab test that would be diagnostic specificity for MPA, absent of circulating ANCA does not exclude the diagnosis of MPA (27).

Renal involvement usually manifests as rapidly progressive glomerulonephritis (GN), which is a hallmark feature of MPA (27). Most common clinical symptoms present as nephrotic range proteinuria, microscopic hematuria, and urinary granular or RBC casts (27).

Pulmonary involvement is seen in $25-55 \%$ of patients (27). It presents as hemoptysis, alveolar hemorrhage, infiltrates, pleural effusion, pulmonary edema, 


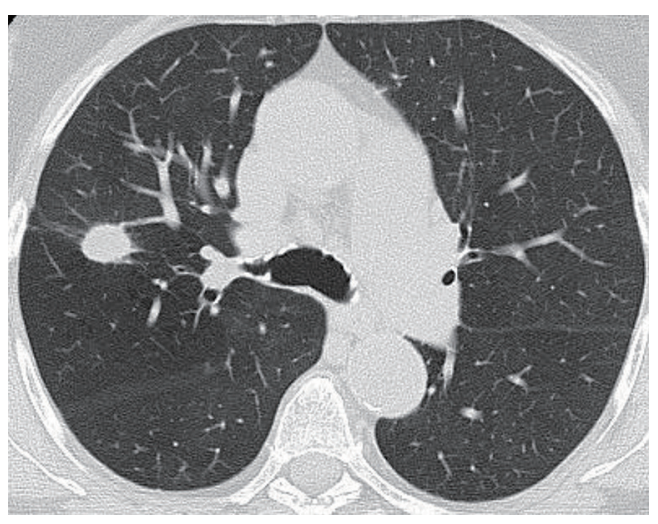

Figure 2 CT chest in 82 years old female with history of sinusitis, shortness of breath and hematuria shows a $15 \mathrm{~mm}$ nodule in the right upper lobe. Biopsy findings were consistent with GPA.

pleuritis and interstitial fibrosis (27). The most common and classic pulmonary manifestation of MPA is diffuse alveolar hemorrhage which is caused by pulmonary capillaritis (27).

Patients usually present with alveolar hemorrhage include dyspnea, cough, hemoptysis, and pleuritic chest pain (27). Chest $\mathrm{X}$-ray and computed tomography are the common modalities used to recognize alveolar hemorrhage (27). Patchy bilateral airspace opacities are seen on CXR, whereas ground glass opacities are seen in $>90 \%$ patients on CT scan corresponding to alveolar hemorrhage (27).

\section{Eosinophilic granulomatous polyangitis (EGPA)}

EGPA is characterized by hypereosinophilia and systemic necrotizing vasculitis seen exclusively in patients with adult onset asthma (28).

Most recently Revised International Chapel Hill consensus conference nomenclature of vasculitides [2012] criteria includes Eosinophil-rich and necrotizing granulomatous inflammation often involving the respiratory tract, and necrotizing vasculitis predominantly affecting small to medium vessel, and associated with asthma and eosinophilia (28).

EGPA commonly involves the lung and skin but can also affect renal, CVS, and GI systems (28). Most confirmatory test is a biopsy of the affected organ which will demonstrate tissue eosinophils with granulomatous reactions (28).

Patients with EGPA most frequently present with asthma. Imaging studies used in patients with EGPA include CXR and CT. CXR would demonstrate pulmonary opacities, and fleeting pulmonary infiltrates that can be rare. CT scan would show parenchymal consolidation with ground glass attenuation (28).

\section{Granulomatosis with polyangiitis (GPA)}

GPA is an uncommon immune mediated systemic small vessel vasculitis characterized by an inflammatory reaction pattern (necrosis, granulomatous inflammation and vasculitis) that occurs in the upper and lower respiratory tracts and kidneys (29). Diagnosis is based on a combination of clinical manifestations positive ANCA serology and histological evidence of necrotizing vasculitis, necrotizing glomerulonephritis or granulomatous inflammation from a relevant organ biopsy, such as skin, lung or kidney (29). Upper respiratory system symptoms are usually present in 90 percent of the patients and lower respiratory tract infections are present in 60 percent (29). Patient present with nasal congestion sinusitis otitis media mastoiditis as well as cough dyspnea and hemoptysis (29).

\section{Imaging modalities}

Chest $\mathrm{CT}$ is more sensitive than chest $\mathrm{x}$-ray. Lesions include infiltrates, nodules, masses, and cavities (29). Lung nodules are the most common manifestation of GPA (Figure 2) and it occurs in $40-70 \%$ of the patients. They can be multiple, bilateral and size varies between $2-4 \mathrm{~cm}$. Cavitation occurs in $25 \%$ of the nodules that are greater than $2 \mathrm{~cm}$ in size. Ground glass opacity and lung consolidation occurs in 30\% of the patients with GPA (29).

\section{Rheumatoid arthritis}

Rheumatoid arthritis is an autoimmune disease of unclear etiology that affects $1 \%$ of population with predisposition in women of age $20-50$ years (30). It is primarily characterized by inflammation and hyperplasia of synovial membrane of symmetric joints and joint destruction, by erosion of cartilage. Rheumatoid Arthritis also cause production of antibodies like rheumatoid factor and anti-citrullinated protein antibody and elevated inflammatory markers ESR and CRP that also help in diagnosing the disease. It is not limited to joints and has systemic features, including cardiovascular, pulmonary, and psychological (30-32). According to revised criteria for diagnosing the disease only joint involvement, blood parameters and duration of symptoms are used for 


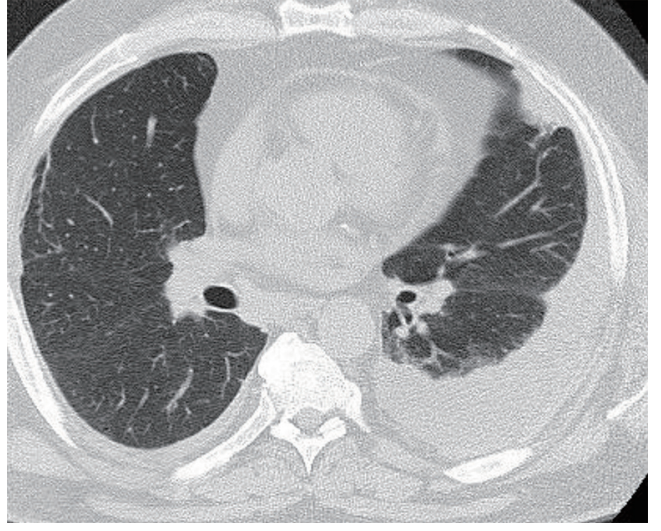

Figure 3 A 54 years old male with history of long standing untreated RA presents with shortness of breath. CT chest shows a pleural based nodule in the lingual and a pleural effusion. Biopsy of the nodule was consistent with rheumatoid necrobiotic nodule.

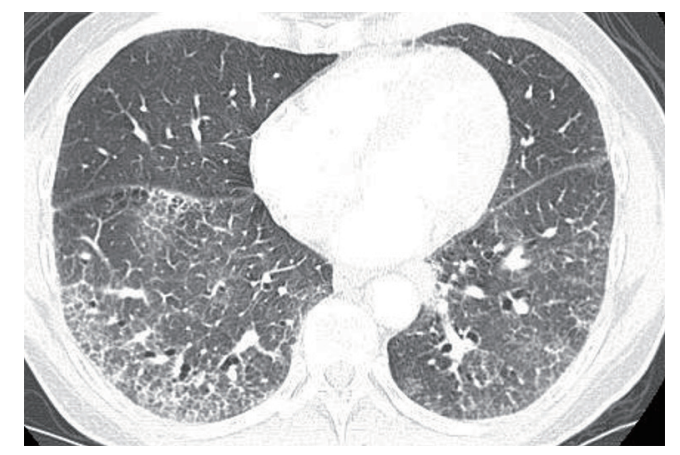

Figure 4 A 58 years old male with history of SLE presents with acute on chronic shortness of breath. CT chest shows multifocal areas of ground-glass opacities in bilateral lower lobes with mild linear interlobular septal thickening, which are suggestive of acute pneumonitis. The posterolateral mosaic appearance of the lungs with reticulations and mild traction bronchiectasis are consistent with fibrotic disease. Overall, imaging findings are consistent with acute on chronic pulmonary SLE.

diagnosis. Radiograph is not part of diagnostic criteria but can be used to monitor disease progression. MRI and ultrasound can be used when diagnosis is not clear. Thoracic complications of Rheumatoid Arthritis are Pleural disease, pleural thickening and pleural effusion. Pleural effusions are usually loculated and unilateral they usually occur late in the disease and associated with Pericarditis and subcutaneous nodules. 2-9\% of patients present with pulmonary fibrosis. On chest radiograph it shows as reticular or reticulonodular pattern and on high-resolution CT scans it shows reticular pattern with irregular interlobular septal thickening. Other thoracic complications include pulmonary nodules (Figure 3) that are similar to subcutaneous nodules and airway disease like bronchiolitis obliterans and bronchiolitis obliterans organizing pneumonia the former shows characteristic mosaic pattern of attenuation and perfusion and later shows air-space consolidation that is usually bilateral and tends to have a patchy peripheral or peribronchial distribution on high-resolution CT (30). According to study done by Tanaka et al. reticulation (98\%) and Ground Glass opacity $(91 \%)$ is the most common CT finding followed by traction bronchiectasis (75\%), architectural distortion (62\%) and honey combing (60\%) (33). Interstitial pneumonia, nonspecific interstitial pneumonia, bronchiolitis, and organizing pneumonia were four predominant CT pattern (33).

\section{Systemic lupus erythematosus (SLE)}

SLE, is an autoimmune disorder which primarily affects women of child bearing age. It can affect any organ but common manifestations include non-deforming arthritis, serositis, photosensitivity and involvement of renal, hematological, and central nervous system (34). SLE is the most common connective tissue disease that affects the lungs (35). The most common pulmonary manifestation of SLE is pleuritis it is present in present in $45-60 \%$ patients of SLE and $5-10 \%$ patient present with pleuritis on initial manifestation (36). 45-60\% patients with pleuritis have pleuritic chest pain. Pleuritis can occur with or without pleural effusion (34). Pleural effusion alone is nonspecific finding on radiology but when present with clinical symptoms of autoimmune disease may suggest SLE (34). It can be differentiated from other causes of pleural effusion by ultrasonographic (US) or CT guided diagnostic thoracentesis (36). Chronic interstitial lung disease is also a common manifestation (Figure 4). Acute lupus pneumonitis and diffuse alveolar hemorrhage are rare complication of SLE (33). The most common HRCT finding in a retrospective study done by Alamoudi et al. were pleural effusion (58\%), consolidation (42\%), atelectasis (42\%) (36). Immunofluorescence of nodule reveal immunoglobins (34). Cardiac disease among patients with SLE is common and can involve the pericardium, myocardium, valves, conduction system, and coronary arteries. Pericarditis is the most common manifestation (30\%) and is usually latent (36). Echocardiography is usually nonspecific for 


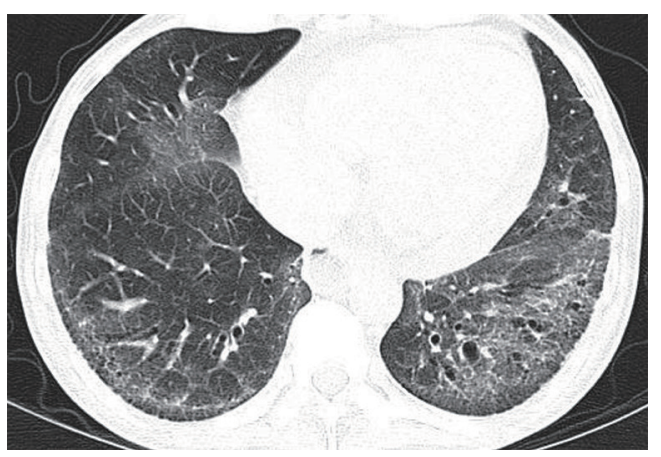

Figure 5 CT chest in a 49 years old male with acute on chronic shortness of breath shows asymmetric multifocal lower lobe predominant interstitial thickening with traction bronchiectasis and scattered ground glass opacities. These imaging findings are consistent with scleroderma related interstitial lung disease with stigmata of fibrosis.

diagnosing pericarditis unless accompanied by pericardial effusion. Clinical correlation and Electrocardiography are helpful in making diagnosis. Contrast enhanced chest CT can also be used (36). Coronary heart disease is one of the rare manifestation of SLE and caused by accelerated atherosclerosis by the use of steroids (36). In patient with accelerated atherosclerosis who can benefit from surgery or intravascular therapy exercise electrocardiography and myocardial scintigraphy may help define reversible ischemia (36).

\section{Scleroderma/crest}

Scleroderma is the disease of unknown etiology; the ratio of disease in women to men is $3: 1$ (35). It is characterized by overproduction of collagen that leads to fibrosis of different organs including lung and skin (35). After international consensus in two subsets of scleroderma emerged after taking into consideration specific nuclear antibodies and capillaroscopy abnormalities (dilation and/ or avascular areas) and specific antinuclear antibodies, diffuse cutaneous SSc (dcSSc) and limited cutaneous SSc (lcSSc) (31). The calcifications, Raynaud's phenomenon, esophageal hypomotility, sclerodactyly, and telangiectasia (CREST) syndrome is also included in (lcSSc) (31). $20-65 \%$ scleroderma patients have pulmonary fibrosis on radiography and is the most common finding. It has basilar predominance and appear as fine reticulation that progress to coarse reticulation and honeycombing (35). The CT features of lung disease in patients with Systemic sclerosis closely resemble nonspecific interstitial pneumonia (NSIP) (Figure 5) $(37,38)$. According to a study done by Schurawitzki et al. when assessing minimal interstitial lung involvement in patients with systemic sclerosis HRCT is much more sensitive than chest radiography (39). In a study done by Owens et al. Chest roentgenograms and pulmonary function were evaluated in 88 patients with CREST syndrome. Pulmonary function was abnormal in $72 \%$. Most common abnormality was isolated decrease in diffusion capacity followed by restrictive abnormalities and airway obstruction (40). Systemic scleroderma can involve myocardium, pericardium and conduction system of the heart (40). Cardiac involvement is one of the poor outcomes of the disease (41). Echocardiography and Doppler echocardiography is the cornerstone of routine heart assessment. For Myocardial perfusion single photon emission computed tomography can be done. Cardiac magnetic resonance imaging can be considered if available (42).

\section{Acknowledgments}

Funding: None.

\section{Footnote}

Provenance and Peer Review: This article was commissioned by the Guest Editor (Ammar Chaudhry) for the series "Role of Precision Imaging in Thoracic Disease" published in Fournal of Thoracic Disease. The article was sent for external peer review organized by the Guest Editor and the editorial office.

Conflicts of Interest: The series "Role of Precision Imaging in Thoracic Disease" was commissioned by the editorial office without any funding or sponsorship. The authors have no other conflicts of interest to declare.

Ethical Statement: The authors are accountable for all aspects of the work in ensuring that questions related to the accuracy or integrity of any part of the work are appropriately investigated and resolved.

Open Access Statement: This is an Open Access article distributed in accordance with the Creative Commons Attribution-NonCommercial-NoDerivs 4.0 International License (CC BY-NC-ND 4.0), which permits the noncommercial replication and distribution of the article with 
the strict proviso that no changes or edits are made and the original work is properly cited (including links to both the formal publication through the relevant DOI and the license). See: https://creativecommons.org/licenses/by-ncnd/4.0/.

\section{References}

1. Collins J, Stern EJ. Chest radiology, the essentials. Lippincott Williams \& Wilkins. (2007) ISBN:0781763142.

2. Miller BH, Rosado-de-christenson ML, Mcadams HP, et al. Thoracic sarcoidosis: radiologic-pathologic correlation. Radiographics 1995;15:421-37.

3. Cozier YC. Assessing the worldwide epidemiology of sarcoidosis: challenges and future directions. Eur Respir J 2016;48:1545-8.

4. Gonzalez-Gay MA, Vazquez-Rodriguez TR, LopezDiaz MJ, et al. Epidemiology of giant cell arteritis and polymyalgia rheumatica. Arthritis Rheum 2009;61:1454.

5. Hunder GG, Bloch DA, Michel BA, et al. The American College of Rheumatology 1990 criteria for the classification of giant cell arteritis. Arthritis Rheum 1990;33:1122-8.

6. Ness T, Bley TA, Schmidt WA, et al. The diagnosis and treatment of giant cell arteritis. Dtsch Arztebl Int 2013;110:376-85.

7. Nuenninghoff DM, Hunder GG, Christianson TJ, et al. Incidence and predictors of large-artery complication (aortic aneurysm, aortic dissection, and/or largeartery stenosis) in patients with giant cell arteritis: a population-based study over 50 years. Arthritis Rheum 2003;48:3522-31.

8. Bienvenu B, Ly KH, Lambert M, et al Groupe d'Étude Français des Artérites des gros Vaisseaux, under the Aegis of the Filière des Maladies Auto-Immunes et Auto-Inflammatoires Rares. Management of giant cell arteritis: Recommendations of the French Study Group for Large Vessel Vasculitis (GEFA). Rev Med Interne 2016;37:154-65.

9. Tomasson G, Peloquin C, Mohammad A, et al. Risk for cardiovascular disease early and late after a diagnosis of giant-cell arteritis: a cohort study. Ann Intern Med 2014;160:73.

10. Hoffman GS. Giant Cell Arteritis. Ann Intern Med 2016;165:ITC65-ITC80.

11. Brugiere O, Mal H, Sleiman C, Groussard O, et al. Isolated pulmonary arteries involvement in a patient with Takayasu's arteritis. Eur Respir J 1998;11:767-70.
12. Mukhtyar C, Guillevin L, Cid MC, et al; European Vasculitis Study Group. EULAR recommendations for the management of large vessel vasculitis. Ann Rheum Dis 2009;68:318-23.

13. Khandelwal N, Kalra N, Garg MK, et al. Multidetector $\mathrm{CT}$ angiography in Takayasu arteritis. Eur J Radiol 2011;77:369-74.

14. Mason JC. Takayasu arteritis--advances in diagnosis and management. Nat Rev Rheumatol 2010;6:406-15.

15. Sharma S, Kamalakar T, Rajani M, et al. The incidence and patterns of pulmonary artery involvement in Takayasu's arteritis. Clin Radiol 1990;42:177-81.

16. Sato O, Cohn DL. Polyarteritis and microscopic polyangiitis. In: Rheumatology, Klippel JH, Dieppe PA (Eds), Mosby, St Louis 2003.

17. Kallenberg CG, Brouwer E, Weening JJ, et al. Antineutrophil cytoplasmic antibodies: current diagnostic and pathophysiological potential. Kidney Int 1994;46:1.

18. Morgan AJ, Schwartz RA. Cutaneous polyarteritis nodosa: a comprehensive review. Int J Dermatol 2010;49:750-6.

19. Stockigt JR, Topliss DJ, Hewett MJ. High-renin hypertension in necrotizing vasculitis. N Engl J Med 1979;300:1218.

20. Kastner D, Gaffney M, Tak T. Polyarteritis nodosa and myocardial infarction. Can J Cardiol 2000;16:515.

21. Findlay AR, Goyal NA, Mozaffar T. An overview of polymyositis and dermatomyositis. Muscle Nerve 2015;51:638-56.

22. Jayakumar D, Zhang R, Wasserman A, et al. Cardiac Manifestations in Idiopathic Inflammatory Myopathies. Cardiol Rev 2019;27:131-7.

23. Hallowell RW, Ascherman DP, Danoff SK. Pulmonary Manifestations of Polymyositis/Dermatomyositis. Semin Respir Crit Care Med 2014;35:239-48.

24. Hozumi H, Fujisawa T, Nakashima R, et al. Comprehensive assessment of myositis-specific autoantibodies in polymyositis/dermatomyositis-associated interstitial lung disease. Respir Med 2016;121:91-9.

25. Marvi U, Chung L, Fiorentino DF. Clinical presentation and evaluation of dermatomyositis. Indian J Dermatol 2012;57:375-81.

26. Ruano CA, Lucas RN, Leal CI, et al. Thoracic Manifestations of Connective Tissue Diseases. Curr Probl Diagn Radiol 2015;44:47-59.

27. Egashira R, Kondo T, Hirai T, et al. CT findings of thoracic manifestations of primary Sjögren syndrome: radiologic-pathologic correlation. Radiographics 2013;33:1933-49. 
28. Flament T, Bigot A, Chaigne B, et al. Pulmonary manifestations of Sjögren's syndrome. Eur Respir Rev 2016;25:110-23.

29. Feragalli B, Mantini C, Sperandeo M, et al. The lung in systemic vasculitis: radiological patterns and differential diagnosis. Br J Radiol 2016;89:20150992.

30. Ananthakrishnan L, Sharma N, Kanne JP. Wegener's granulomatosis in the chest: high-resolution CT findings. AJR Am J Roentgenol 2009;192:676-82.

31. Mayberry JP, Primack SL, Müller NL. Thoracic manifestations of systemic autoimmune diseases: radiographic and high-resolution CT findings. Radiographics 2000;20:1623-35.

32. McInnes IB, Schett G. The pathogenesis of rheumatoid arthritis. N Engl J Med 2011;365:2205-19.

33. Tins BJ, Butler R. Imaging in rheumatology: reconciling radiology and rheumatology. Insights Imaging 2013;4:799-810.

34. Tanaka N, Kim JS, Newell JD, et al. Rheumatoid arthritis-related lung diseases: CT findings. Radiology 2004;232:81-91.

35. Lalani TA, Kanne JP, Hatfield GA, et al. Imaging findings in systemic lupus erythematosus. Radiographics 2004;24:1069-86.

36. Alamoudi OS, Attar SM. Pulmonary manifestations in

Cite this article as: Gul M, Moinuddin S, Alam A, Aftab I, Shah Z, Chaudhry A. Thoracic imaging finding of rheumatic diseases. J Thorac Dis 2020;12(9):5110-5118. doi: 10.21037/ jtd.2020.04.16 systemic lupus erythematosus: association with disease activity. Respirology 2015;20:474-80.

37. Hachulla E, Launay D. Diagnosis and classification of systemic sclerosis. Clin Rev Allergy Immunol 2011;40:78-83.

38. Desai SR, Veeraraghavan S, Hansell DM, et al. CT features of lung disease in patients with systemic sclerosis: comparison with idiopathic pulmonary fibrosis and nonspecific interstitial pneumonia. Radiology 2004;232:560-7.

39. Schurawitzki H, Stiglbauer R, Graninger W, et al. Interstitial lung disease in progressive systemic sclerosis: high-resolution CT versus radiography. Radiology 1990;176:755-9.

40. Owens GR, Fino GJ, Herbert DL, et al. Pulmonary function in progressive systemic sclerosis: comparison of CREST syndrome variant with diffuse scleroderma. Chest 1983;84:546-50.

41. Deswal A, Follansbee WP. Cardiac involvement in scleroderma. Rheum Dis Clin North Am 1996;22:841-60.

42. Meune C, Vignaux O, Kahan A, et al. Heart involvement in systemic sclerosis: evolving concept and diagnostic methodologies. Arch Cardiovasc Dis 2010;103:46-52. 